Received: 17.11 .2019

Revised: 30.04 .2020

Accepted: 19.06 .2020

DOI: $10.17804 / 2410-9908.2020 .3 .006-018$

\title{
DETERMINING THE MOMENT OF DESTRUCTION OF PROTECTIVE COATINGS ON PIPES AND SPHERICAL VESSELS
}

\author{
V. V. Struzhanov ${ }^{1, \text { a }^{*}}$ and A. E. Chaikin ${ }^{2, \text { b) }}$ \\ ${ }^{1}$ Institute of Engineering Science, Ural Branch of the Russian Academy of Sciences, \\ 34 Komsomolskaya St., Ekaterinburg, 620049, Russian Federation \\ ${ }^{2}$ Institute of Natural Sciences and Mathematics, B. N. Yeltsin Ural Federal University, \\ 48 Kuibysheva St., Ekaterinburg, 620000, Russian Federation \\ a) iD http://orcid.org/0000-0002-3669-2032 stru@imach.uran.ru; \\ б) iD http://orcid.org/0000-0001-5582-2384 chaykin.ae@yandex.ru \\ *Corresponding author: E-mail: stru@imach.uran.ru \\ Address for correspondence: 34 Komsomolskaya St., 620049, Ekaterinburg, Russian Federation \\ Tel.: +7(343)362-30-19
}

An analytical method is developed to determine the moment of destruction of thin coatings on pipes and spherical vessels. The coating material works at the stage of elasticity; it has the property of strain softening, that is, destruction with increasing deformation occurs in the process of stress drop. The properties of the coating material are described by convex-concave potentials both under uniaxial tension and in a plane stress state. To determine the moment of destruction, methods of the mathematical theory of catastrophes are applied, which allow one to find all the equilibrium positions of systems and the point of loss of stability of the deformation process.

Keywords: thin coating, pipe, spherical vessel, curves of equilibrium states, loss of stability, destruction, Lamé problem.

\section{References}

1. Fedorov Yu.Yu., Popov S.N., Savvina A.V., Vasilyev S.V., Rodionov A.K. Evaluation of the Axial Stresses of a Gas Pipeline Made of Reinforced Polyethylene Pipes under Conditions of Permafrost Soils. Diagnostics, Resource and Mechanics of materials and structures, 2017, iss. 3, pp. 36-41. DOI: 10.17804/2410-9908.2017.3.036-041. URL: http://dream-journal.org/issues/ 2017-3/2017-3_122.html (assessed: 22.03.2018).

2. $\quad$ Struzhanov V.V., Mironov V.I. Deformatsionnoe razuprochnenie materiala $v$ elementakh konstruktsiy [Strain Softening of Material in Structural Elements]. Ekaterinburg, UrO RAN Publ., 1995, $190 \mathrm{p}$.

3. Andrasic C.P., Parker A.P. Dimensionless stress intensity factors for cracked thick cylinders under polynomial crack face loadings. Engng. Fract. Mech., 1984, vol. 19, no. 1, pp. 187-193.

4. Shannon R.W.E. Stress intensity factors for thick-walled cylinders. Int. J. Pres. Ves. and Piping, 1974, vol. 2, pp. 19-29.

5. Kachanov L.M. Osnovy teorii plastichnosti [Fundamentals of the Theory of Plasticity]. Moscow, Nauka Publ., 1969, 420 p. (In Russian).

6. Korkin A.V., Struzhanov V.V., Chaykin A.E. Stability of uniform tension of a disk with a central zone of softening material. In: Proceedings of the Eleventh All-Russian Scientific Conference with International Participation "Mathematical Modeling and Boundary Value Problems" (May, 27-30, 2019, Samara, Russian Federation), vol. 1, Samara State Technical Univ., Samara, 2019, pp. 64-68. (In Russian). 
7. Struzhanov V.V., Korkin A.V., Chaykin A. E. One approach to determination of the ultimate load-bearing capacity of mechanical systems with softening elements. Vestn. Samar. Gos. Tekhn. Univ., Ser. Fiz.-Mat. Nauki [J. Samara State Tech. Univ., Ser. Phys. Math. Sci.], 2018, vol. 22 (4), pp. 762-773. DOI: 10.14498/vsgtu1624. (In Russian).

8. Struzhanov V.V. The determination of the deformation diagram of a material with a falling branch using the torsion diagram of a cylindrical sample. Sib. Zh. Ind. Mat., 2012, vol. 15 (1), pp. 138-144. (In Russian).

9. Poston T., Stuart I. Teoriya katastroph i ee prilozheniya [Poston T., Stewart I. Catastrophe Theory and Its Applications, London, San Francisco, Melbourne, Pitman, 1978]. Moscow, Mir Publ., 1980, 608 p. (In Russian).

10. Gilmore R. Prikladnaya teoriya katastroph. Book 1 [Gilmore R. Catastrophe Theory for Scientists and Enqincers, New York, Dover, 1993]. Moscow, Mir Publ., 1984, 350 p. (In Russian). 11. Timoshenko S.P., Gudier D.N. Teoriya uprugosti [Timoshenko S.P., Goodier J.N. Theory of Elasticity, New York, Toronto, London, McGraw Hill Book Company Inc., 1951]. Moscow, Nauka Publ., 1979, 560 p. (In Russian).

12. Pars L. Analiticheskaya dinamika [Pars L. A Treatise on Analytical Dynamics. Heinemann, London, 1965; reprinted by Ox Bow Press, Woodbridge, CT, USA, 1979]. Moscow, Nauka Publ., 1971, $636 \mathrm{p}$. 
Подана в журнал: 17.11.2020

УДК 539.3

DOI: $10.17804 / 2410-9908.2020 .3 .006-018$

\title{
ОБ ОПРЕДЕЛЕНИИ МОМЕНТА РАЗРУШЕНИЯ ЗАЩИТНЫХ ПОКРЫТИЙ ТРУБ И СФЕРИЧЕСКИХ СОСУДОВ
}

\author{
В. В. Стружанов ${ }^{1,)^{*}}$, А. Е. Чайкин ${ }^{2, \text { б) }}$ \\ ${ }^{1}$ Федеральное государственное бюджетное учреждение науки \\ «Институт машиноведения Уральского отделения Российской академии наук», \\ ул. Комсомольская 34, 620049, г. Екатеринбург, Российская Федерация \\ ${ }^{2}$ Институт естественных наук и математики Уральского Федерального университета», \\ ул. Мира 19, 620000, г. Екатеринбург, Российская Федерация. \\ a) iD http://orcid.org/0000-0002-3669-2032 stru@imach.uran.ru; \\ б) (iD http://orcid.org/0000-0001-5582-2384 chaykin.ae@yandex.ru \\ *Ответственный автор. Электронная почта: stru@imach.uran.ru \\ Адрес для переписки: ул. Комсомольская, 34, г. Екатеринбург, Российская Федерация \\ Тел.: +7(343)362-30-19
}

Разработан аналитический метод определения момента разрушения тонких покрытий труб и сферических сосудов, материал которых работает на стадии упругости. Материал покрытий обладает свойством деформационного разупрочнения, то есть разрушение при возрастающей деформации происходит в процессе падения напряжений. Свойства материала покрытия описываются выпукло-вогнутыми потенциалами как при одноосном растяжении, так и при плоском напряженном состоянии. Для определения момента разрушения используются методы математической теории катастроф, которые позволяют найти все положения равновесия систем и точку потери устойчивости процесса деформирования.

Ключевые слова: тонкое покрытия, труба, сферический сосуд, кривые равновесных состояний, потеря устойчивости, разрушение, задача Ляме.

\section{1. Введение}

Цилиндрические и сферические элементы конструкций часто используются в технике. Это резервуары различного назначения, трубопроводы, котлы, корпуса двигателей. Разработка методов расчета составных цилиндров на прочность становится актуальной и с появлением новых современных материалов [1]. При этом ставятся задачи прогнозирования разрушения материалов и гарантирование работы ответственных конструкций [2-4].

Для повышения работоспособности и жизнедеятельности таких элементов конструкций часто используют защитные покрытия, которые ограждают их от вредного влияния агрессивных сред и от воздействия на окружающую среду, когда трубы и сферические сосуды выполнены из токсичных материалов. В процессе возрастания внутреннего давления защитные покрытия могут разрушиться и перестать выполнять свои функции. Следовательно, задача о прогнозировании их разрушения является актуальной. В данной работе предложена методика расчета момента разрушения покрытий. Она основана на применении методов математической теории катастроф при том условии, что свойства материалов покрытий описываются выпукло-вогнутыми потенциалами, отражающими их упрочнение и разупрочнение, а также возможность разрушения. 


\section{2. Механические системы}

Будем рассматривать две механические системы. Первая - это толстостенная длинная труба с внутренним радиусом, равным $a$, и внешним, равным $b$, имеющая тонкое покрытие толщиной $t$. Вторая - толстостенная сфера (внутренний радиус $a$, внешний $-b$ ), которая также покрыта тонкой оболочкой (толщина $t$ ). Толстостенные элементы всегда находятся в состоянии упругости. Свойства упругого материала определяют модуль Юнга $E$ и коэффициент Пуассона $v$. Покрытия изготовлены из материала, который перед разрушением проходит стадию разупрочнения. В упругости материал покрытий имеет модуль Юнга $H$ и коэффициент Пуассона $\mu$. Труба и сфера находятся под действием квазистатически возрастающего внутреннего давления интенсивностью $p$ (мягкое нагружение). Либо точкам внутренних границ задается квазистатически возрастающее радиальное перемещение $w$ (жесткое нагружение). При нагружении точки внешних границ трубы и сферы из-за наличия покрытий получают некоторые, заранее неизвестные, радиальные перемещения $v$. Нагружения происходят при постоянной температуре. Для каждой механической системы требуется найти значение параметров $w$ или $p$, при достижении которых покрытия разрушаются.

\section{3. Свойство покрытий}

Определим свойства материала покрытий на всех стадиях деформирования, включая и стадию разупрочнения. При пропорциональном нагружении для расчета напряженнодеформированного состояния, как правило, используется теория малых упруго-пластических деформаций [5]. Однако ее первое положение о постоянстве объемного модуля на стадии разупрочнения не выполняется в силу наличия объемного разрушения материала. Вместо второго положения о пропорциональности девиаторов тензоров напряжений и деформаций будем сохранять пропорциональность полных тензоров напряжений и деформаций с некоторым переменным матричным коэффициентом, зависящим от деформаций. Это означает, что в области неупругости линии уровня функции потенциальной энергии подобны линиям уровня в области упругости. В случае плоско-напряженного состояния имеем [6, 7]:

$$
\left(\frac{\sigma_{1}}{\sigma_{2}}\right)=\psi\left(\varepsilon_{1}, \varepsilon_{2}\right) \cdot \frac{H}{1-\mu^{2}} \cdot\left(\begin{array}{cc}
1 & \mu \\
\mu & 1
\end{array}\right) \cdot\left(\begin{array}{l}
\varepsilon_{1} \\
\varepsilon_{2}
\end{array}\right) .
$$

Для определения параметра $\psi$ воспользуемся полной диаграммой с падающей ветвью на стадии разупрочнения при чистом сдвиге [8]. Пусть $\tau=G \cdot\left(\gamma-50 \gamma^{2}\right)$. Здесь $\tau$ - касательное напряжение, $\gamma$ - сдвиг, $G=\frac{H}{2 \cdot(1+\mu)}-$ модуль сдвига в упругости. Тогда энергия деформаций $V=\int_{0}^{\gamma} \tau d \gamma=V^{e} \cdot\left(1-\frac{100}{3} \cdot \sqrt{\frac{2 \cdot V^{e}}{G}}\right), V^{e}=\frac{1}{2} \cdot G \gamma^{2}, \gamma=\sqrt{\frac{2 \cdot V^{e}}{G}}$.

Энергия упругой деформации в общем случае плоско-напряженного состояния

$$
V^{e}\left(\varepsilon_{1}, \varepsilon_{2}\right)=H \cdot\left[2 \cdot\left(1-\mu^{2}\right)\right]^{-1} \cdot\left(\varepsilon_{1}^{2}+2 \mu \varepsilon_{1} \varepsilon_{2}+\varepsilon_{2}^{2}\right)
$$

Здесь $\varepsilon_{1}, \varepsilon_{2}-$ компоненты плоской деформации в прямоугольной декартовой системе координат.

Так как $\sigma_{1}=\frac{d V}{d \varepsilon_{1}}, \sigma_{2}=\frac{d V}{d \varepsilon_{2}}$, то из равенства (1) находим, что 


$$
\psi=1-100 \cdot \sqrt{V^{e} \cdot \frac{1+\mu}{H}} .
$$

\section{4. Разрушение покрытия трубы (жесткое нагружение)}

Для определения момента разрушения покрытия в системе (упругая труба - покрытие) при постепенном возрастании параметра $w$ будем использовать аппарат математической теории катастроф $[9,10]$. Его применение основано на построении лагранжиана системы, который в силу равенства нулю кинетической энергии представляет сумму потенциальных энергий деформаций элементов системы. Очевидно, что он зависит от параметра управления $w$ (задаваемая величина) и состояния $v$ (обобщенная координата).

Энергию деформаций трубы найдем, вычисляя напряжения и деформации из решения осесимметричной задачи Ляме [11] с граничными условиями $\left.u\right|_{r=a}=w,\left.u\right|_{r=b}=v,(u-$ радиальные перемещения точек трубы). Производя необходимые действия, получаем

$$
\begin{gathered}
\varepsilon_{r}=\frac{1}{b^{2}-a^{2}} \cdot\left[(b v-w a)-\frac{1}{r^{2}} a b \cdot(w b-v a)\right], \\
\varepsilon_{\theta}=\frac{1}{b^{2}-a^{2}} \cdot\left[(b v-w a)+\frac{1}{r^{2}} a b \cdot(w b-v a)\right], \\
\sigma_{r}=\frac{E}{\left(1-v^{2}\right) \cdot\left(b^{2}-a^{2}\right)} \cdot\left[(b v-w a) \cdot(1+v)-\frac{1}{r^{2}} a b \cdot(w b-v a) \cdot(1-v)\right], \\
\sigma_{\theta}=\frac{E}{\left(1-v^{2}\right) \cdot\left(b^{2}-a^{2}\right)} \cdot\left[(b v-w a) \cdot(1+v)+\frac{1}{r^{2}} a b \cdot(w b-v a) \cdot(1-v)\right] .
\end{gathered}
$$

Здесь $\varepsilon_{r}, \varepsilon_{\theta}, \sigma_{r}, \sigma_{\theta}$ - соответственно радиальные и тангециальные деформации и напряжения.

Энергия элемента материала равна:

$$
\Pi=\frac{1}{2} \cdot\left(\sigma_{r} \varepsilon_{r}+\sigma_{\theta} \varepsilon_{\theta}\right)
$$

тогда полная энергия деформаций трубы:

$$
W_{1}=L \int_{0}^{2 \pi b} \int_{a}^{b} \Pi \cdot r d r d \theta=\frac{\pi E}{b^{2}-a^{2}}\left[\frac{(b v-w a)^{2}}{1-v}+\frac{(w b-v a)^{2}}{1+v}\right] \cdot L .
$$

( $L$ - длина трубы).

В цилиндрической оболочке (покрытии) имеются только деформации $\varepsilon_{\theta}=\frac{v}{b}$, в упругости $-\sigma_{\theta}=\frac{H}{1-\mu^{2}} \varepsilon_{\theta}, V^{e}=\frac{1}{2} \cdot \frac{H}{1-\mu^{2}} \varepsilon_{\theta}^{2}$. Тогда, используя равенства (1) и (2), находим: $\psi=1-100 \cdot \varepsilon_{\theta} \sqrt{\frac{1}{2(1-\mu)}}, \sigma_{\theta}=\frac{H}{1-\mu^{2}} \varepsilon_{\theta} \cdot\left(1-100 \cdot \varepsilon_{\theta} \sqrt{\frac{1}{2(1-\mu)}}\right)$. 
Отсюда энергия покрытия:

$$
W_{2}=2 \pi b t L \int_{0}^{\varepsilon_{\theta}} \sigma_{\theta} d \varepsilon_{\theta}=2 \pi b t L \cdot \frac{H}{1-\mu^{2}} \cdot\left(\frac{\varepsilon_{\theta}^{2}}{2}-\frac{100}{3} \cdot \varepsilon_{\theta}^{3} \sqrt{\frac{1}{2(1-\mu)}}\right) \text {. }
$$

( $2 \pi b t L$ - объем покрытия).

Итак, лагранжиан системы есть сумма $W=W_{1}+W_{2}$, которая есть функция от неизвестного параметра состояния v. Теперь, используя уравнение Лагранжа второго рода [12], получаем уравнение равновесия системы:

$$
\frac{d W}{d v}=\frac{2 \pi E}{b^{2}-a^{2}}\left[\frac{b(b v-w a)}{1-v}-\frac{a(w b-v a)}{1+v}\right] L+2 \pi b t L \frac{H}{1-\mu^{2}}\left(\frac{v}{b^{2}}-100 \frac{v^{2}}{b^{3}} \sqrt{\frac{1}{2(1-\mu)}}\right)=0 .
$$

Решая уравнение (4), находим зависимость $w$ от $v$, т. е. функцию $w=f(v)$.

После разрушения покрытия внешняя поверхность трубы уже свободна от действия внешних сил. Решим задачу Ляме с граничными условиями $\left.u\right|_{r=a}=w, \sigma_{r=b}=0$. В результате находим связь между $w$ и $v$ :

$$
w=k v, \quad k=\left[(1+v) b^{2}+(1-v) a^{2}\right] \cdot(2 a b)^{-1} .
$$

Решая уравнение $f(v)=k v$, находим значение $v=v^{*}$, которое определяет точку пересечения кривой $f(v)$ и прямой (5). Эта точка $A$ с координатами $\left(v^{*}, w^{*}=f\left(v^{*}\right)\right)$ (рис. 1$)$. Очевидно, что уже при $v>v^{*}$ положения равновесия системы определяются точками прямой (5) (рис. 1, прямая 2). Следовательно, при $v>v$ * покрытие разрушается. Отметим также, что на отрезке $0 \leq v \leq v^{*}$ имеем $\frac{d w}{d v}>0$, т. е. имеет место монотонное возрастание функции $w=f(v)$. Таким образом, деформирование идет равновесно вплоть до момента разрушения покрытия.

В качестве примера произведем вычисления при значениях $t=1 \mathrm{mм}, a=3 \mathrm{Mм}$, $b=10$ мм, $E=0,16 \cdot 10^{5}$ МПа, $v=0,3$ (свинец), $H=2 \cdot 10^{5} \mathrm{MПа,} \mu=0,3$ (сталь). В результате получаем кривую $w=f(v)$ (рис. 1, кривая 1) и прямую $w=k v$ (рис. 1, прямая 2). Точка их пересечения имеет координаты $\left(v^{*}=0,237\right.$ мм, $w^{*}=0,598$ мм). Так как кривая 1 на участке $0 \leq v \leq 0,23$ мм монотонно возрастает, то процесс деградации механических свойств покрытия происходит равновесно вплоть до разрушения в точке $A$ (рис. 1 ). 


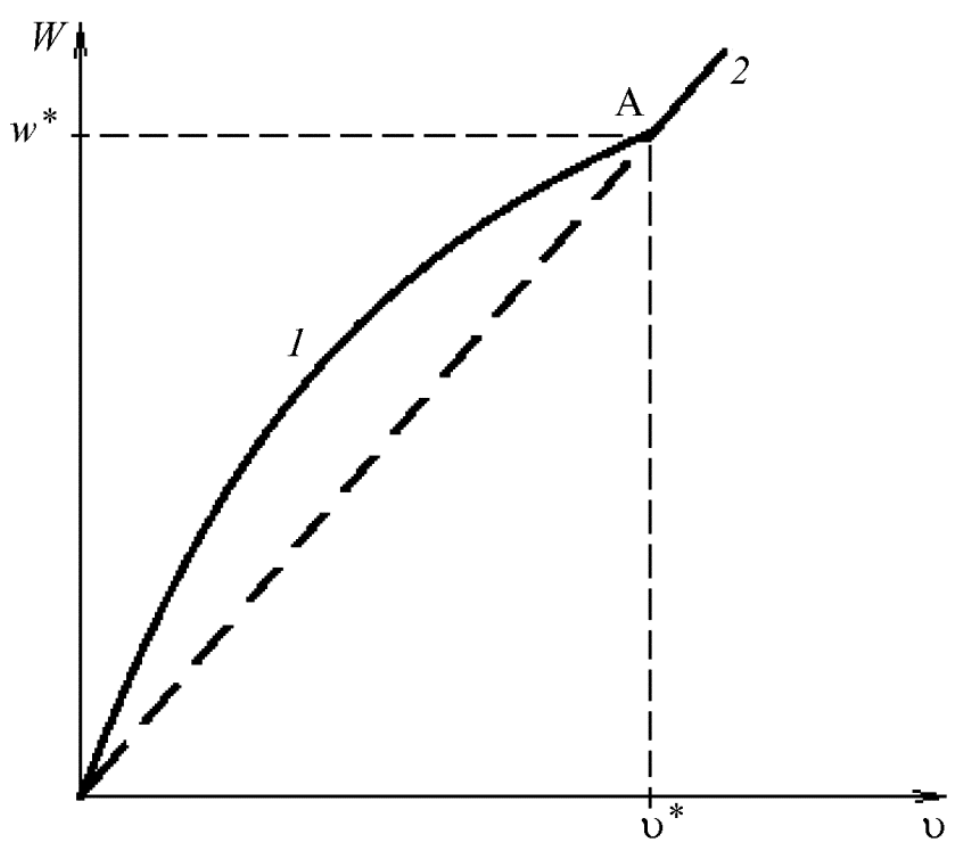

Рис. 1. Качественный вид кривой равновесных состояний (линия 1, переходящая в линию 2 после разрушения) при жестком нагружении системы

\section{5. Разрушение покрытия трубы (мягкое нагружение)}

Построим лагранжиан данной системы. Сначала найдем решение задачи Ляме с граничными условиями $\left.u\right|_{r=b}=v, \sigma_{r=a}=-p$ ( $p$ - внутреннее давление). Получаем:

$$
\begin{gathered}
\varepsilon_{r}=M-\frac{1}{r^{2}} N, \varepsilon_{\theta}=M+\frac{1}{r^{2}} N ; \\
\sigma_{r}=\frac{E}{1-v^{2}} \cdot\left[M(1+v)-\frac{N}{r^{2}}(1-v)\right], \\
\sigma_{\theta}=\frac{E}{1-v^{2}} \cdot\left[M(1+v)+\frac{N}{r^{2}}(1-v)\right],
\end{gathered}
$$

где

$$
\begin{gathered}
N=b^{2} A B, \quad M=\left(\frac{v}{b}-A B\right), \\
A=\frac{a^{2}}{(1-v) b^{2}+a^{2}(1+v)}, B=\frac{p b\left(1-v^{2}\right)+E v(1+v)}{b E} .
\end{gathered}
$$

Тогда энергия трубы

$$
\Pi_{1}=L \int_{0}^{2 \pi} \int_{a}^{b} \frac{1}{2}\left(\sigma_{r} \varepsilon_{r}+\sigma_{\theta} \varepsilon_{\theta}\right) r d r d \theta=L \cdot \frac{E \pi\left(b^{2}-a^{2}\right)}{1-v^{2}}\left[M^{2}(1+v)+\frac{1}{b^{2} a^{2}} N^{2}(1-v)\right]=
$$




$$
=\frac{L E \pi\left(b^{2}-a^{2}\right)}{1-v^{2}}\left[\frac{v^{2}}{b^{2}}(1+v)-\frac{a^{2}\left[p b\left(1-v^{2}\right)+E v(1+v)\right] \cdot\left[v E(1+v)-p b\left(1-v^{2}\right)\right]}{\left[(1-v) b^{2}+a^{2}(1+v)\right] b^{2} E^{2}}\right] .
$$

В лагранжиан входит энергия покрытия, которая задана формулой (3), а также работа внешней силы, взятая со знаком минус [12], т. е.

$$
\Pi_{2}=-\left.p \cdot 2 \pi a L u\right|_{r=a}
$$

Из решения задачи Ляме имеем:

$$
\left.u\right|_{r=a}=\left(\frac{v}{b}-A B\right) a-\frac{1}{a} b^{2} A B
$$

Итак лагранжиан системы равен $\Pi=\Pi_{1}+W_{2}+\Pi_{2}$. Тогда уравнение равновесия:

$$
\begin{aligned}
& \frac{d \Pi}{d v}=\frac{L E \pi\left(b^{2}-a^{2}\right) 2 v}{(1-v) b^{2}+a^{2}(1+v)}-2 \pi a L p\left(\frac{a}{b}+\frac{a v(1+v)\left(b^{2}-a^{2}\right)}{\left[(1-v) b^{2}+a^{2}(1+v)\right] b}\right)+ \\
& +2 \pi b t L \frac{H}{1-\mu^{2}}\left(\frac{v}{b}-100 \frac{v^{2}}{b^{3}} \sqrt{\frac{1}{2(1-\mu)}}\right)=0 .
\end{aligned}
$$

Из уравнения (6) получаем зависимость $p$ от $v$ (функцию $p=\varphi(v))$.

Теперь решаем задачу Ляме для трубы без покрытия с граничными условиями $\left.\sigma_{r}\right|_{r=a}=-p,\left.\sigma_{r}\right|_{r=b}=0$. В результате получаем зависимость

$$
p=h v, h=E\left(b^{2}-a^{2}\right)\left(2 a^{2} b\right)^{-1}
$$

Решение $v=v^{*}$ уравнения $\varphi(v)=h v$ определяет точку с координатами $\left(v^{*}, p^{*}=\varphi\left(v^{*}\right)\right)-$ точку пересечения кривой $\varphi(v)$ и прямой $h v$, после достижения которой уже точки прямой отвечают равновесным состояниям системы, т. е. трубы с разрушенным покрытием. В отличие от предыдущего случая в отрезке $0 \leq v \leq v^{*}$ кривая равновесных состояний системы $p=\varphi(v)$ имеет максимум при некотором значении $v \leq v^{\prime} \leq v^{*}\left(\left.\frac{d p}{d v}\right|_{v=v^{\prime}}=0\right)$. Поэтому при постепенном возрастании параметра $p$ при $p \geq p^{*}$ система имеет уже несколько возможных положений равновесия. Однако согласно принципу промедления [9], система сначала проходит положения равновесия, отвечающие точкам восходящей ветви функции $p=\varphi(v)$. После достижения значения $p^{\prime}=\varphi\left(v^{\prime}\right)$ равновесие становится неустойчивым и малейшее возрастание параметра $p$ приводит к скачкообразному переходу в новое устойчивое равновесие, которое отвечает некоторой точке прямой $h v$. Таким образом, имеет место скачкообразное (катастрофическое) разрушение покрытия.

Произведем вычисления при тех же значениях, что и в предыдущем пункте. Получаем кривую $p=\varphi(v)$ (рис. 2, кривая 1) и прямую $p=h v$ (рис. 2, прямая 2). Точка их пересечения имеет координаты $\left(v^{*}=0,119 \mathrm{Mм}, p^{*}=950 \mathrm{MПа}\right)$. Максимальное значение $p=p^{\prime}=980 \mathrm{MПа}$ $\left(v^{\prime}=0,105\right.$ мм). При увеличении $p>p^{\prime}$ система скачком переходит в новое устойчивое равновесие, отвечающее точке $\mathrm{C}$ прямой $p=h v$. Следовательно, реализуется катастрофическое разрушение покрытия. 


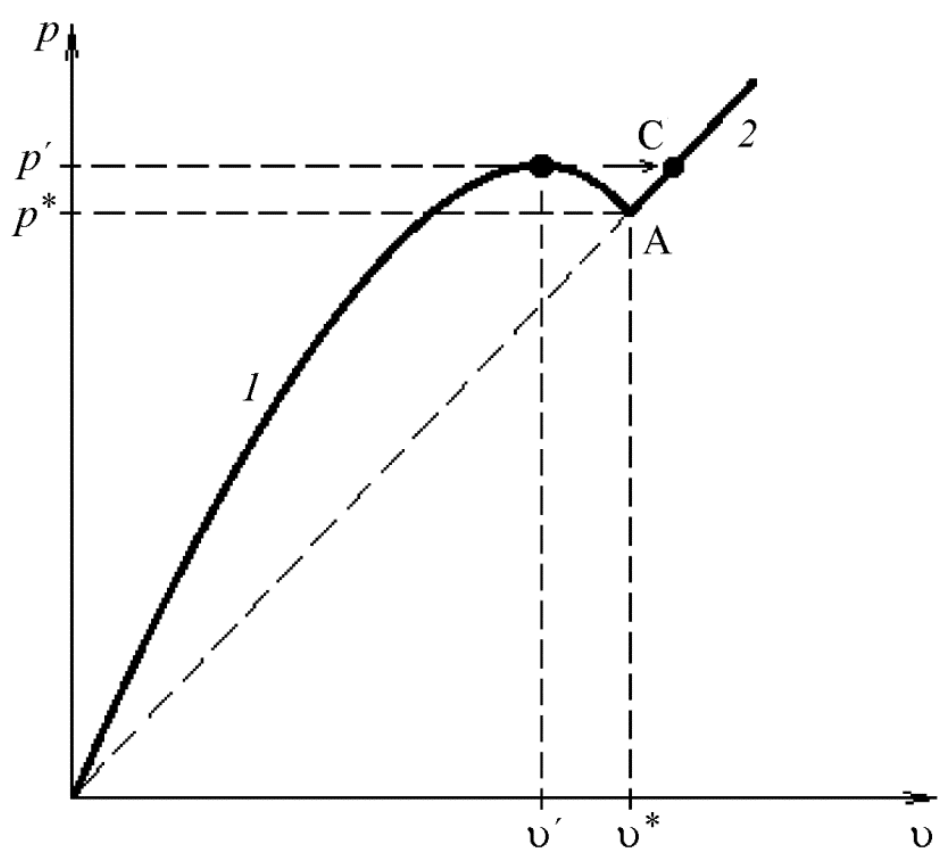

Рис. 2. Качественный вид кривой равновесных состояний

(линия 1, переходящая в линию 2 после разрушения) при мягком нагружении системы

\section{6. Разрушение покрытия сферического сосуда (жесткое нагружение)}

Построим лагранжиан системы при задании точкам внутренней поверхности перемещения $w$. Он состоит из суммы энергий толстостенной сферы и покрытия. Сначала решим задачу Ляме для толстостенной сферы (центрально симметричная задача) [11] при граничных условиях $\left.u\right|_{r=a}=w,\left.u\right|_{r=b}=v(u-$ радиальное перемещение).

$$
\begin{gathered}
\varepsilon_{r}=\frac{1}{b^{3}-a^{3}}\left[\left(v b^{2}-w a^{2}\right)-\frac{2}{r^{3}} b^{2} a^{2}(w b-v a)\right] \\
\varepsilon_{\theta}=\varepsilon_{\varphi}=\frac{1}{b^{3}-a^{3}}\left[\left(v b^{2}-w a^{2}\right)+\frac{1}{r^{3}} b^{2} a^{2}(w b-v a)\right], \\
\sigma_{r}=\frac{E}{b^{3}-a^{3}}\left[\frac{v b^{2}-w a^{2}}{1-2 v}-\frac{2 b^{2} a^{2}(w b-v a)}{r^{3}(1+v)}\right], \\
\sigma_{\theta}=\sigma_{\varphi}=\frac{E}{b^{3}-a^{3}}\left[\frac{v b^{2}-w a^{2}}{1-2 v}+\frac{b^{2} a^{2}(w b-v a)}{r^{3}(1+v)}\right],
\end{gathered}
$$

энергия сферы равна

$$
W_{s}=\int_{-\pi 0}^{\pi} \int_{0}^{\pi} \int_{a}^{b} \frac{1}{2}\left(\sigma_{r} \varepsilon_{r}+2 \sigma_{\theta} \varepsilon_{\theta}\right) \cdot r^{2} \sin \theta d r d \theta d \varphi=\frac{2 \pi E}{b^{3}-a^{3}}\left[\frac{\left(v b^{2}-w a^{2}\right)^{2}}{1-2 v}+\frac{2 b a(w b-v a)^{2}}{1+v}\right] .
$$

Здесь $\varepsilon_{r}, \varepsilon_{\theta}, \varepsilon_{\varphi}-$ радиальные и тангенциальные деформации; $\sigma_{r}, \sigma_{\theta}, \sigma_{\varphi}-$ радиальные и тангенциальные напряжения в толстостенной сфере. 
В сферической оболочке (покрытии) имеют место только деформации $\varepsilon_{\theta}=\varepsilon_{\varphi}=\frac{v}{b}=\varepsilon$ (равномерное двухосное растяжение). Тогда из формулы (2) находим: $\psi=1-100 \varepsilon \sqrt{\frac{1+\mu}{1-\mu}}$. Отсюда $\sigma_{\theta}=\sigma_{\varphi}=\sigma=\left(1-100 \varepsilon \sqrt{\frac{1+\mu}{1-\mu}}\right) \frac{H}{1-\mu} \varepsilon$ (это следует из формулы (1)). Энергия элемента покрытия:

$$
\frac{1}{2}\left(\sigma_{\theta} \varepsilon_{\theta}+\sigma_{\varphi} \varepsilon_{\varphi}\right)=\left(1-100 \varepsilon \sqrt{\frac{1+\mu}{1-\mu}}\right) \frac{H \varepsilon}{1-\mu} \varepsilon=\left(1-100 \frac{v}{b} \sqrt{\frac{1+\mu}{1-\mu}}\right) \frac{H}{1-\mu} \frac{v^{2}}{b^{2}}
$$

Общая энергия покрытия

$$
V_{s}=4 \pi b^{2} t\left(1-100 \frac{v}{b} \sqrt{\frac{1+\mu}{1-\mu}}\right) \frac{H}{1-\mu} \frac{v^{2}}{b^{2}},
$$

(4 $\pi b^{2} t$ - объем покрытия).

Таким образом, энергия всей системы $U=W_{s}+V_{s}$. Здесь $w-$ параметр управления (задаваемая величина); $v$ - параметр состояния (определяемая величина). Тогда имеем уравнение равновесия:

$$
\frac{d U}{d v}=\frac{4 \pi E}{b^{3}-a^{3}}\left[\frac{\left(v b^{2}-w a^{2}\right) b^{2}}{1-2 v}-\frac{2 b a^{2}(w b-v a)}{1+v}\right]+4 \pi t \frac{2 H v}{1-\mu}\left(1-\frac{150}{b} v \sqrt{\frac{1+\mu}{1-\mu}}\right)=0 .
$$
$w=\psi(v)$.

Из уравнения (8) получаем связь между $w$ и $v$ в состоянии равновесия, т. е. функцию

Теперь определим связь между $w$ и $v$ при отсутствии покрытия. Для этого решим задачу Ляме для сферы с условиями на границе $\left.u\right|_{r=a}=w,\left.\sigma_{r}\right|_{r=b}=0$. В результате получаем зависимость

$$
w=q v, q=\left[(1+v) b^{3}+2(1-2 v) a^{3}\right] \cdot\left[3 a^{2} b(1-v)\right]^{-1} .
$$

Решение $v=v^{*}$ уравнения $\psi(v)=q v$ определяет $\left(v^{*}, w^{*}=\psi\left(v^{*}\right)\right)-$ точку пересечения кривой $\psi(v)$ и прямой (9). Так как $\frac{d w}{d v}>0$ на отрезке $0 \leq v \leq v^{*}$, то разрушение покрытия происходит после достижения параметром $w$ значения $w^{*}$. До этого момента деформирование системы происходит равновесно. Численный расчет при тех же механических характеристиках материалов на стадии упругости, что и в предыдущих случаях, а также, считая $t=1$ мм, $a=3 \mathrm{мм}, b=10$ мм, получаем $v^{*}=0,05$ мм, $w^{*}=0,34$ мм. Кривая равновесных состояний системы подобна кривой, изображенной на рис. 1.

\section{7. Разрушение покрытия сферического сосуда (мягкое нагружение)}

Снова строим лагранжиан системы, состоящий теперь из суммы энергий сферы, покрытия и работы, реализующих внутреннее давление внешних сил, взятой со знаком минус. 
Энергию деформаций сферы определяем, используя решение задачи Ляме для центрально симметричного деформирования толстостенной сферы при граничных условиях $\left.u\right|_{r=b}=v,\left.\sigma_{r}\right|_{r=a}=-p$. Имеем:

$$
\begin{gathered}
\varepsilon_{r}=\frac{1}{E M}\left((1-2 v) N-\frac{2}{r^{3}} a^{3} b^{2}(1+v) K\right), \\
\varepsilon_{\varphi}=\varepsilon_{\theta}=\frac{1}{E M}\left((1-2 v) N+\frac{1}{r^{3}} a^{3} b^{2}(1+v) K\right), \\
\sigma_{r}=\frac{1}{M}\left(N-\frac{2}{r^{3}} a^{3} b^{2} K\right), \\
\sigma_{\varphi}=\sigma_{\theta}=\frac{1}{M}\left(N+\frac{1}{r^{3}} a^{3} b^{2} K\right) .
\end{gathered}
$$

Здесь

$$
\begin{gathered}
M=a^{3}(1+v)+2 b^{3}(1-2 v), \\
N=2 E v b^{2}-p a^{3}(1+v), \\
K=p b(1-2 v)+E v .
\end{gathered}
$$

Теперь энергия деформаций сферы равна

$$
W_{s}^{p}=4 \pi \frac{E}{M^{2}}\left(b^{3}-a^{3}\right)\left[\frac{1}{2}(1-2 v) N^{2}+a^{3} b(1+v) K^{2}\right] .
$$

Энергия внешних сил, взятая со знаком минус:

$$
Q=-\left.4 \pi a^{2} p u\right|_{r=a}=-\left.4 \pi a^{2} p\left(\varepsilon_{\theta} r\right)\right|_{r=a}=-4 \pi a^{2} p \frac{1}{E M}\left[a(1-2 v) N+a b^{2}(1+v) K\right]
$$

Таким образом полная энергия системы $U_{p}=W_{s}^{p}+V_{s}+Q$ и уравнение равновесия имеет вид:

$$
\begin{aligned}
& \frac{d U_{p}}{d v}=\frac{8 \pi E^{2}}{M^{2}}\left(b^{3}-a^{3}\right)\left[(1-2 v) b^{2} N+a^{3} b(1+v) K\right]-12 \pi(1-v) p \frac{a^{3} b^{2}}{M}+ \\
& +4 \pi t \frac{2 H v}{1-\mu}\left(1-\frac{150}{b} v \sqrt{\frac{1+\mu}{1-\mu}}\right)=0 .
\end{aligned}
$$

Из уравнения (10) находим связь между параметрами $p$ и $v$ в состоянии равновесия, т. е. функцию $p=\beta(v)$. 
Далее определяем связь между $p$ и $v$ при отсутствии покрытия. Для этого решаем задачу Ляме для сферы с граничными условиями $\left.\sigma_{r}\right|_{r=a}=-p,\left.\sigma_{r}\right|_{r=b}=0$. В результате получаем зависимость:

$$
p=m v, m=2 E\left(b^{3}-a^{3}\right)\left[3(1-v) b a^{3}\right]^{-1} .
$$

Решение $v=v^{*}$ уравнения $\beta(v)=m v$ определяет $\left(v^{*}, p^{*}=\beta\left(v^{*}\right)\right)$ - точку пересечения кривой $\beta(v)$ и прямой (11). После этой точки исходящая из нее прямая (11) отвечает уже равновесным состояниям сферы с разрушенным покрытием. На участке $0 \leq v \leq v$ * кривая равновесных состояний системы $p=\beta(v)$ имеет максимум при некотором значении $v=v^{\prime}<v^{*}$. Поэтому при постепенном возрастании параметра $p$ при $p>p *$ система имеет уже несколько положений равновесия. Согласно принципу промедления [9] система сначала проходит положения равновесия, отвечающие точкам восходящей ветви функции $p=\beta(v)$. При достижении значения $p=p^{\prime}=\beta\left(v^{\prime}\right)$ происходит скачкообразный переход в новое устойчивое положение равновесия, которое отвечает некоторой точке прямой тv. В результате реализуется динамическое разрушение покрытия.

Численный расчет для приведенных выше данных дает координаты точки пересечения кривой $p=\beta(v)$ и прямой (11) $v^{*}=0,049$ мм; $p^{*}=270$ МПа. Максимальное значение внутреннего давления, при котором реализуется скачкообразное разрушение покрытия, равно $p^{\prime}=283$ МПа $\left(v^{\prime}=0,04\right.$ мм). Качественный вид кривой равновесных состояний подобен кривой, изображенной на рис. 2.

\section{8. Заключение}

Сформулирована задача о разрушении защитных покрытий труб и сферических сосудов. Покрытия выполнены из разупрочняющихся материалов. На основе использования методов математической теории катастроф произведен расчет предельных параметров нагружения, при которых происходит разрушение покрытий.

\section{Литература}

1. Evaluation of the Axial Stresses of a Gas Pipeline Made of Reinforced Polyethylene Pipes under Conditions of Permafrost Soils / Yu. Yu. Fedorov, S. N. Popov, A. V. Savvina, S. V. Vasilyev, A. K. Rodionov // Diagnostics, Resource and Mechanics of materials and structures. 2017. - Iss. 3. - P. 36- 41. - DOI: 10.17804/2410-9908.2017.3.036-041. - URL: http://dreamjournal.org/issues/2017-3/2017-3_122.html (assessed: 22.03.2018).

2. Стружанов В. В., Миронов В. И. Деформационное разупрочнение материала в элементах конструкций. - Екатеринбург : УрО РАН, 1995. - 190 с.

3. Andrasic C. P., Parker A. P. Dimensionless stress intensity factors for cracked thick cylinders under polynomial crack face loadings // Engng. Fract. Mech. - 1984. - Vol. 19. - P. 187-193.

4. Shannon R. W. E. Stress intensity factors for thick-walled cylinders // Int. J. Pres. Ves. and Piping. - 1974. - Vol. 2. - P. 19-29.

5. Качанов Л. М. Основы теории пластичности / пер. с англ. - М. : Наука, 1969. - 420 с.

6. Коркин А. В., Стружанов В. В., Чайкин А. Е. Об устойчивости равномерного растяжения диска с центральной зоной из разупрочняющегося материала // Математическое моделирование и краевые задачи, Самара, 27-30 мая, 2019: материалы 11 Всероссийской научной конференции с международным участием. - Том 1. - Самара : СамГТУ, 2019. - С. 64-69.

7. Стружанов В. В., Коркин А. В., Чайкин А. Е. Об одном подходе к определению предельной несущей способности механических систем с разупрочняющимися элементами // Вестн. Сам. гос. техн. ун-та. Сер. Физ.-мат. науки. - 2018. - Т. 22, № 4. -С. 762-773. DOI: $10.14498 /$ vsgtu 1624. 
8. Стружанов В. В. Определение диаграммы деформирования с падающей ветвью по диаграмме кручения цилиндрического образца // Сибирский журнал индустриальной математики. - 2012. - Т. XV, № 1 (49). - С. 138-144.

9. Постон Т., Стюарт И. Теория катастроф и ее приложения / пер. с англ. - М. : Мир, 1980. $-608 \mathrm{c}$.

10. Гилмор Р. Прикладная теория катастроф. Кн. 1 / пер. с англ. - М. : Мир, 1984. - 350 с. 11. Тимошенко С. П., Гудьер Дж. Теория упругости / пер. с англ. - М. : Наука, 1979. $560 \mathrm{c}$.

12. Парс Л. Аналитическая динамика / пер. с англ. - М. : Наука, 1971. - 636 с. 